\title{
ASO Author Reflections: Developing Next-Generation Intraperitoneal Chemotherapy
}

\author{
Abdelkader Taibi, MD, $\mathbf{P h D}^{1,2}$ (D) , Olivia Sgarbura, $\mathrm{MD}, \mathrm{PhD}^{3}$, and Martin Hübner, $\mathrm{MD}, \mathrm{PhD}^{4}$ \\ ${ }^{1}$ Digestive Surgery Department, Dupuytren Limoges University Hospital, Limoges, France; ${ }^{2}$ University Limoges, CNRS, \\ XLIM, UMR 7252, Limoges, France; ${ }^{3}$ Surgical Oncology Department, Montpellier Cancer Institute (ICM), University of \\ Montpellier, Montpellier, France; ${ }^{4}$ Department of Visceral Surgery, Lausanne University Hospital (CHUV), University of \\ Lausanne (UNIL), Lausanne, Switzerland
}

\section{PAST}

Peritoneal metastases (PM) of different origins remain a diagnostic and therapeutic challenge, with still disappointing outcomes when treated with palliative chemotherapy alone. The last decades have witnessed the validation of complex treatment protocols incorporating both modern combination chemotherapies and cytoreductive surgeries and leading to highly improved survival for patients with PM. The addition of hyperthermic intraperitoneal chemotherapy (HIPEC) was initially received with reluctance but, later, succeeded in gaining wider acceptance. ${ }^{1}$ However, it still remains controversial due to conflicting results from very recent pivotal randomized trials (PRODIGE7, OVHIPEC, PROPHYLOCHIP, and COLOPEC). Further limitations are of a pharmacological nature (single-shot administration, low tissue penetration, heterogeneous distribution) and concern regarding the large polymorphism of treatment protocols and indications.

\section{PRESENT}

Pressurized intraperitoneal aerosol chemotherapy (PIPAC) is a novel mode for intraperitoneal drug delivery, improving, at least in theory, these pharmacokinetic limitations: aerosol for better distribution, pressure to enhance

(C) Society of Surgical Oncology 2020

First Received: 2 November 2020

Accepted: 2 November 2020;

Published Online: 20 November 2020

A. Taibi, $\mathrm{MD}, \mathrm{PhD}$

e-mail: abdelkader.taibi@hotmail.Fr tissue penetration, and repeated minimally invasive treatment. ${ }^{2}$ Its feasibility, safety, and favorable tolerance profile have already been convincingly demonstrated, and preliminary data on efficacy were encouraging. ${ }^{2}$ In addition, standardized training modules have helped to maintain common standards for technique, indications, and treatment protocols. ${ }^{3}$ The addition of electrostatic precipitation (ePIPAC) has been proposed by the pioneering team (unpublished), claiming accelerated and improved distribution and tissue uptake and hence allowing the administration time to be safely reduced. ${ }^{4}$ So far, because of the unavailability of data, few centers have started to explore the use of ePIPAC, and unfortunately with varying protocols. ${ }^{3,4}$

Our recent analysis confirms the safe use of ePIPAC in the largest cohort of patients to date, with surrogate outcomes for efficacy comparable to the standard PIPAC protocol. $^{5}$

\section{FUTURE}

Potential improvements of the current PIPAC protocol concern not only the prospective inclusion of electrostatic precipitation but also optimization of other pertinent variables such as the exposure time, temperature, pressure, drugs and their concentration, carrier solution, and number of treatments. In addition, further research is needed to evaluate its combination with systemic chemotherapy (alternate or concomitant) and/or cytoreductive surgery. Some lessons should have been learned from the HIPEC experience. Exploring PIPAC requires: (1) preclinical optimization before widespread use in human and ambitious phase III trials, (2) coordinated multicenter collaborative work to define research priorities and take 
advantage of synergies, and (3) a common standard to avoid heterogeneity of treatment protocols. The IDEAL framework turned out to be a useful guide for the limited number of leading academic centers. ${ }^{3}$ The obtained results are very likely not only to help PIPAC but also to serve for standardization and refinement of current HIPEC and catheter-based IP protocols, the three pillars of next-generation IP chemotherapy.

DISCLOSURES AT, OS, MH none.

\section{REFERENCES}

1. Morano WF, Khalili M, Chi DS, Bowne WB, Esquivel J. Clinical studies in CRS and HIPEC: Trials, tribulations, and future directions-A systematic review. $J$ Surg Oncol. févr 2018;117(2):245-59.

2. Alyami M, Hübner M, Grass F, Bakrin N, Villeneuve L, Laplace $\mathrm{N}$, et al. Pressurised intraperitoneal aerosol chemotherapy: rationale, evidence, and potential indications. Lancet Oncol. 2019;20(7):e368-77.

3. Sgarbura O, Villeneuve L, Alyami M, Bakrin N, Torrent JJ, Eveno $\mathrm{C}$, et al. Current practice of pressurized intraperitoneal aerosol chemotherapy (PIPAC): Still standardized or on the verge of diversification? Eur J Surg Oncol. 29 août 2020;

4. Van de Sande L, Rahimi-Gorji M, Giordano S, Davoli E, Matteo C, Detlefsen S, et al. Electrostatic intraperitoneal aerosol delivery of nanoparticles: proof of concept and preclinical validation. $A d v$ Healthc Mater. 2020;9(16):e2000655.

5. Taibi A, Teixeira Farinha H, Durand Fontanier S, Sayedalamin Z, Hübner M, Sgarbura O. Pressurized intraperitoneal aerosol chemotherapy enhanced by electrostatic precipitation (ePIPAC) for patients with peritoneal metastases. Ann Surg Oncol. 2020. https://doi.org/10.1245/s10434-020-09332-6.

Publisher's Note Springer Nature remains neutral with regard to jurisdictional claims in published maps and institutional affiliations. 